\title{
La aplicación del programa "escritura creativa" y su influencia en el desarrollo de la capacidad de producción de textos narrativos en niños de educación primaria
}

The application of the program" creative writing "and its influence on the development of the ability to produce narrative texts in primary school children

Vilma Doris Huamaní Alejo ${ }^{1}$

\section{RESUMEN}

Objetivo: Determinar la influencia del programa "Escritura Creativa" en el desarrollo de la capacidad de producción de textos narrativos en los estudiantes del $4^{\circ}$ grado de la Institución Educativa de Primaria $\mathrm{N}^{\circ}$ 22238 Chincha, 2015. Metodos: La investigacion fue de tipo Cuasi experimental, es decir, que se tomó dos grupos intactos con pre y post test. Para ello se diseñó un programa experimental Escritura creativa. La muestra estuvo conformada por un grupo experimental que corresponde a la sección "B" y el grupo de control a la sección "A" cada sección cuenta con 25 estudiantes de ambos sexos con una categoría intelectual normal y seleccionada mediante el método de carácter no probabilístico intencional. Resultado: Hay evidencias suficientes con $p=0,000$ para afirmar que existe un logro significativo en la capacidad de produccion de terxtos narrativos donde los resultados del grupo experimental obtuvieron mejores resultados con un promedio $=36,92$ puntos, estos fueron estadísticamente superiores al grupo de control con un promedio $=24,84$ puntos, lográndose mejorar en 12,08 puntos. Conclusiones: La aplicación del programa escritura creativa mejoró en las dimensiones de la presentación textual, en el proceso de la escritura y en el uso de la normativa por lo que se recomienda al ministerio de educación para su aplicación en las instituciones educativas de nivel primaria porque son relatos sobre la base de las experiencias centradas de quien escribe y propicia un aprendizaje significativo del enfoque comunicativo textual.

Palabras clave: Programa "Escritura creativa", producción de textos narrativos.

\section{ABSTRACT}

Objective: Determine the influence of the program "Creative Writing" in the development of the capacity of production of narrative texts in the students of the 4th grade of the Educational Institution of Primary No. 22238 Chincha, 2015. Methods: The research was of the quasi-experimental type, that is to say, who took two intact groups with pre and post test. For this an experimental program Creative Writing was designed. The sample consisted of an experimental group corresponding to section "B" and the control group to section "A" each section has 25 students of both sexes with a normal intellectual category and selected by the non-probabilistic method intentional. Result: There is sufficient evidence with $p=0.000$ to affirm that there is a significant achievement in the production capacity of narrative terxtos where the results of the experimental group obtained better results with an average $=36.92$ points, these were statistically superior to the control group with an average $=24.84$ points, achieving an improvement of 12.08 points. Conclusions: The application of the creative writing program improved in the dimensions of the textual presentation, in the writing process and in the use of the regulations, for which its application is recommended, in the educational institutions of primary level because they favor the ease of the writing of narrative texts, because they are stories based on the centered experiences of the writer.

Keywords: program "Creative writing", production of narrative texts. 


\section{INTRODUCCIÓN}

En este proceso de investigación pedagógica el problema que se abordó está relacionado con la implicancia que tiene la escritura creativa en la producción de textos narrativos donde se observó grandes dificultades en la producción de textos: como el desconocimiento de las características de los textos narrativos, falencias en la coherencia y cohesión, en el uso adecuado de los signos de puntuación, las frases que deben utilizarse en cada momento, etc. frente a esta situación tuve la necesidad de realizar una investigación que se centra en su carácter de experimento. En efecto, el programa "Escritura creativa" pretende desencadenar procesos cognitivos, afectivos y sociales a través de los cuales los estudiantes, ponen de manifiesto sus sentimientos y pensamientos ante los demás aprovechando la imaginación, fantasía y en especial las historias reales presentes en los estudiantes del cuarto grado de primaria.

Gallego y García (2010), en su investigación: La comprensión y producción de textos narrativos en el grado tercero del Instituto técnico superior. La intervención se realizó con 30 niños y niñas del grado tercero del turno tarde, donde recojo una de sus conclusiones que tuvo más relevancia: Las competencias lectoras y escritoras no son innatas en los seres humanos, éstas también se dan a través de un proceso de construcción y de interacción de los humanos, en este caso los estudiantes con los escritos de ciertos autores propios para su edad, y de otros escritos propios de los niños de su edad.

De dicha investigación se puede concluir que el estudiante se interrelaciona con su espacio, su contexto para facilitar su creación en la producción de textos narrativos y que estos sean más subjetivas que necesariamente no deben seguir un modelo sino una guía de imaginación propias de sus vivencias.

Campos y Mariños (2009) en su tesis titulada Influencia del programa "Escribe cortito, pero bonito" en la producción de textos narrativos: mitos, cuentos y leyendas del área de comunicación de los estudiantes de cuarto grado de primaria de la I. E. № 88005 "Corazón de Jesús" de Chimbote - Ancash, 2009" concluye que su programa contribuyó a fortalecer en los estudiantes la capacidad de producción de textos, prestando atención a la forma y contenido, utilizando la lengua de forma adecuada a la situación y al contexto en un estilo propio.

El individuo creativo es una persona que resuelve problemas con regularidad, elabora productos o define cuestiones nuevas en un campo, de un modo que al principio es considerado nuevo, pero que al final llega a ser aceptado en un contexto cultural concreto (Gardner, 1995). Lomas (1999) afirma que la escritura es un proceso muy diferente del acto de hablar, siendo la diferencia principal entre hablar y escribir que el hablar es sumamente interactivo y al escribir solo interactuamos con un trozo de papel o un monitor. Además, agrega que las estrategias de escritura creativa es un método sencillo pero eficaz que nos ayudará a sumar opciones para alcanzar nuestros objetivos con la escritura. Beaugrande (1981) indica que los textos se producen a través de operaciones complejas que son guiadas por la memoria, la atención, el control motor, el recuerdo y la motivación.

Cassany, (2003) señala que la palabra texto significa cualquier manifestación verbal y completa que se produzca en una comunicación". Por tanto, son textos, los escritos de literatura, las redacciones de los alumnos, las exposiciones de los alumnos, las exposiciones del profesor, los diálogos y las conversaciones de los alumnos en el aula o en el patio, las noticias de la prensa, la publicidad, etc. Los textos pueden ser orales o escritos; literarios o no; para leer o escuchar, o para decir o escribir. Asimismo, Cassany (1990), menciona que sólo hay un camino posible para escribir: buscar experiencias que impliquen emocionalmente a las personas de los alumnos; usar lo escrito para explorar su mundo personal: lo que les gusta, interesa o preocupa. La escritura extensiva (diarios íntimos, libros de notas o cuadernos de reflexión y aprendizaje...) es la mejor actividad para este fin. Se trata de llevar un cuaderno en el que el alumno anota periódicamente, a iniciativa propia y sin intervención del maestro, las experiencias diarias que más le interesen (sensaciones, ideas, intereses...). Se valora la cantidad de escritura (más que la calidad), su valor epistemológico (por encima del comunicativo); y se propone desarrollar buenas actitudes hacia la escritura: crear confianza, consolidar hábitos, formar buenas opiniones, etc. Además, el cuaderno constituye una materia prima de primera calidad para elaborar después otros textos.

En la presente investigación se plantea el objetivo de Determinar la influencia del programa "Escritura Creativa" en el desarrollo de la capacidad de producción de textos narrativos en los estudiantes del $4^{\circ}$ grado de la Institución Educativa de Primaria $\mathrm{N}^{\circ} 22238$ Chincha. Mejorando sus capacidades de expresión y comprensión de textos, desarrollar la imaginación, fantasía e historias reales de los niños, y replicar estrategias didácticas contenidas en el programa experimental "escritura creativa", en otras instituciones educativas del Perú, generando con ello un efecto multiplicador. 


\section{MATERIAL Y MÉTODOS}

La investigación se realizó en la Institución Educativa N$^{\circ} 22238$ de la ciudad de Chincha, Perú, durante el año 2015. La muestra de estudio estuvo constituida por niños y niñas del $4^{\circ}$ grado de educación primaria, siendo un total de 100 estudiantes. Se uso la muestra de carácter no probabilística, intencionada, debido a la accesibilidad e interés por verificar el efecto del programa experimental en dicho grado de estudios.

Tomándose en cuenta dos secciones "A" y "B" asignándole el rol de grupo experimental a la sección "B" y el grupo de control a la sección "A". La investigación se ejecutó tomando en consideración el Diseño Experimental de clase Cuasi experimental, de dos grupos intactos (grupo experimental y grupo de control) con pre y post test. Donde se evaluó a ambos grupos la variable dependiente, y a uno de ellos se aplicó el tratamiento experimental mientras el otro tratamiento sigue con las actividades rutinarias. La metodología empleada se muestra a continuación:

$\begin{array}{lccc}\text { Tratamientos } & \begin{array}{c}\text { Pre } \\ \text { experimental }\end{array} & \begin{array}{c}\text { Aplicación } \\ \text { de } \\ \text { programa }\end{array} & \begin{array}{c}\text { Post } \\ \text { experimental }\end{array} \\ \begin{array}{l}\text { Grupo } \\ \text { experimental }\end{array} & \mathrm{O} 1 & \mathrm{Si} & \mathrm{O} 2 \\ \begin{array}{l}\text { Grupo } \\ \text { control }\end{array} & \mathrm{O} 3 & \mathrm{No} & \mathrm{O} 4 \\ \end{array}$

Dónde:

O1 y O3: observaciones pre experimentales

$\mathrm{O} 2$ y O4: observaciones post experimentales

En relación al Método, se utilizó, el experimento, es decir que se diseñó y valido un programa experimental en el que se organicen las estrategias del programa de Escritura creativa. Concurrentemente se utilizó la técnica de observación sistemática, lo cual permitió el diseño y validación de un instrumento de recolección de datos que provengan del experimento y que se aplicará antes y después del experimento. Cuyo instrumento se recurrió al juicio de expertos y para verificar la confiabilidad se recurrió a la prueba Alpha de Cronbach.

\section{Pre Test}

Institución Educativa:
Grado: $\quad$ Fecha:

Género:

Número de orden:

Tema:

Cantidad de Hojas

\begin{tabular}{|c|c|c|c|c|}
\hline $\begin{array}{c}\text { No } \\
\text { hay }\end{array}$ & $\begin{array}{c}\text { Muy } \\
\text { poco }\end{array}$ & Regular & Bueno & Muy bueno \\
\hline $0 \%$ & $1 \%-25 \%$ & $25 \%-50 \%$ & $51 \%-75 \%$ & $75 \%-100 \%$ \\
\hline & & & & \\
\hline
\end{tabular}

\section{Puntaje}

\begin{tabular}{lc}
\hline 1 & No hay \\
\hline $\mathbf{2}$ & Muy poco \\
\hline $\mathbf{3}$ & Regular \\
\hline $\mathbf{4}$ & Bueno \\
\hline $\mathbf{5}$ & Muy bueno \\
\hline
\end{tabular}

Suma el puntaje total y se multiplica por 0,2 el puntaje obtenido se compara con la Tabla:

Tabla de Nivel de Producción de textos:

\begin{tabular}{lc} 
Muy bajo & $0-10$ \\
\hline Bajo & $11-13$ \\
\hline Regular & $14-15$ \\
\hline Alto & $16-17$ \\
\hline Muy alto & $18-20$ \\
\hline
\end{tabular}




\begin{tabular}{|c|c|c|c|c|c|}
\hline & $\begin{array}{l}\text { बे } \\
\frac{\text { C }}{Z} \\
\text { Z }\end{array}$ & $\begin{array}{l}\text { O } \\
\text { O } \\
\text { Ż } \\
\text { ż }\end{array}$ & 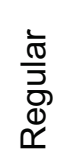 & 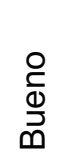 & 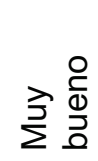 \\
\hline \multicolumn{6}{|l|}{ Presentación del texto } \\
\hline 1. En la portada incluye título y autor & & & & & \\
\hline 2. La portada cuenta con ilustración & & & & & \\
\hline 3. Hay Legitimidad de la letra & & & & & \\
\hline $\begin{array}{l}\text { 4. Las ilustraciones tienen relación con cada etapa del tema } \\
\text { del texto }\end{array}$ & & & & & \\
\hline 5. La presentación es impecable y agradable & & & & & \\
\hline 6. Tiene buena distribución de espacios & & & & & \\
\hline \multicolumn{6}{|l|}{ Proceso de la escritura } \\
\hline 7. El título va de acuerdo al tema & & & & & \\
\hline 8. Aparece claramente el inicio, desarrollo y desenlace & & & & & \\
\hline 9. Se ubica en el tiempo & & & & & \\
\hline 10. Está claramente expresado el conflicto o problema & & & & & \\
\hline 11. Aparecen los personajes y el lugar & & & & & \\
\hline 12. Utiliza un vocabulario amplio y no repite palabras & & & & & \\
\hline 13. Los hechos tienen un orden secuencial & & & & & \\
\hline 14. La figura central se describe al detalle & & & & & \\
\hline Uso de la normativa & & & & & \\
\hline 15. No tiene errores ortográficos & & & & & \\
\hline 16. Utiliza adecuadamente los signos de puntuación & & & & & \\
\hline 17. Las oraciones están bien estructuradas & & & & & \\
\hline 18. Uso adecuado de las mayúsculas & & & & & \\
\hline $\begin{array}{l}\text { 19. Utiliza a lo menos } 3 \text { conectores que destacan las } \\
\text { relaciones semánticas }\end{array}$ & & & & & \\
\hline 20. Uso adecuado de los tiempos verbales & & & & & \\
\hline
\end{tabular}

\section{RESULTADOS}

Los estudiantes del grupo experimental fueron mejores en escritura creativa y la producción de textos (promedio $=36,92$ puntos) después de la aplicación del programa Escritura Creativa respecto a los estudiantes del grupo control (promedio $=24,84$ puntos), lográndose mejorar en 12,08 puntos (Tabla 1).

Tabla 1. Diferencias de medias de las variables escritura creativa y la producción de textos narrativos.

\begin{tabular}{lccc} 
& \multicolumn{2}{c}{ Grupo } & $\begin{array}{c}\text { Test U de } \\
\text { Estadístico }\end{array}$ \\
\cline { 2 - 4 } & Control $(n=25)$ & Experimental $(n=25)$ & \\
\hline Media & 24,80 & Pre test & $\mathrm{Z}=-0,653$ \\
Desv. Tip & 4,62 & 26,04 & $\mathrm{p}=0,514$ \\
\hline & & 5,41 & \\
\hline Media & 24,84 & Post test & $\mathrm{Z}=-4,569$ \\
Desv. Tip & 3,14 & 36,92 & $\mathrm{p}=0,000$ \\
\hline
\end{tabular}

Los estudiantes del grupo experimental fueron mejores en la dimensión presentación textual (promedio $=11,04$ ) después de la aplicación del programa Escritura Creativa respecto a los estudiantes del grupo de control (promedio $=7,04$ ). Lográndose mejorar en 4 puntos (Tabla 2). 
Tabla 2. Diferencia de medias de control y experimental según la dimensión Presentación textual.

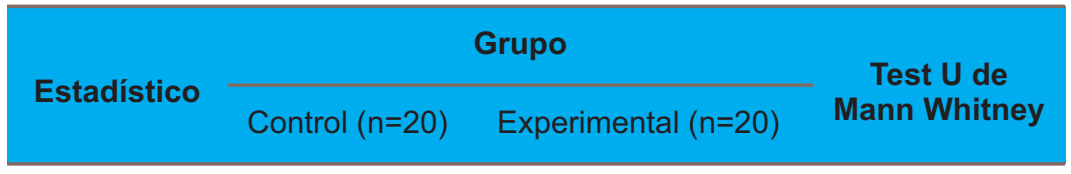

\begin{tabular}{lccc}
\multicolumn{3}{c}{ Pre test } \\
\hline Media & 7,32 & 7,88 & $\mathrm{Z}=-0,945$ \\
Desv. Tip & 1,03 & 1,76 & $\mathrm{p}=0,345$ \\
\hline \multicolumn{3}{c}{ Post test } \\
\hline Media & 7,04 & 11,04 & $\mathrm{Z}=-4,807$ \\
Desv. Tip & 1,37 & 3,89 & $\mathrm{p}=0,000$ \\
\hline
\end{tabular}

Los estudiantes del grupo experimental fueron mejores en el Proceso de escritura (promedio $=15,40$ ) después de la aplicación del programa
Escritura Creativa respecto a los estudiantes del grupo de control (promedio $=10,36$ ). Lográndose mejorar en 5,04 puntos (Tabla 3).

Tabla 3. Diferencia de medias del grupo de control y experimental según la dimensión Proceso de escritura.

\begin{tabular}{lccc} 
& \multicolumn{2}{c}{ Grupo } & $\begin{array}{c}\text { Test U de } \\
\text { Estadístico }\end{array}$ \\
\cline { 2 - 4 } & Control $(n=20)$ & Experimental $(n=20)$ & Mann Whitney \\
Media & 10,40 & 10,48 & $\mathrm{Z}=-0,724$ \\
Desv. Tip & 3,29 & 2,34 & $\mathrm{p}=0,469$ \\
\hline & & Post test & \\
\hline Media & 10,36 & 15,40 & $\mathrm{Z}=-4,406$ \\
Desv. Tip & 1,68 & 4,85 & $\mathrm{p}=0,000$ \\
\hline
\end{tabular}

Los estudiantes del grupo experimental fueron mejores en el uso de la normativa (promedio $=14,60)$ después de la aplicación del programa
Escritura Creativa respecto a los estudiantes del grupo de control (promedio $=6,97$ ). Lográndose mejorar en 7,63 puntos (Tabla 4).

Tabla 4. Diferencia de medias del grupo de control y experimental según la dimensión uso de la normativa

\begin{tabular}{|c|c|c|c|}
\hline \multirow{2}{*}{ Estadístico } & \multicolumn{2}{|r|}{ Grupo } & \multirow{2}{*}{$\begin{array}{c}\text { Test U de } \\
\text { Mann Whitney }\end{array}$} \\
\hline & Control $(n=20)$ & Experimental $(n=20)$ & \\
\hline \multicolumn{4}{|c|}{ Pre test } \\
\hline Media & 7,08 & 7,68 & $Z=-0,801$ \\
\hline Desv. Tip & 1,66 & 2,04 & $p=0,423$ \\
\hline \multicolumn{4}{|c|}{ Post test } \\
\hline Media & 6,97 & 14,60 & $Z=-4,139$ \\
\hline Desv. Tip & 1,68 & 3,56 & $p=0,000$ \\
\hline
\end{tabular}




\section{DISCUSIÓN}

Podemos observar que en el trabajo de investigación de Fuentes (2008), estudio realizado sobre "Un modelo de intervención didáctica del aula", revela que la intervención didáctica, a través del modelamiento meta cognitivo, permite generar en los estudiantes un cambio en el procesamiento y organización de la ideas, como también, auto regular el proceso de producción textual, con la aplicación de las estrategias de aprendizaje adquiridas durante el desarrollo de la intervención, por otro lado sumamos a esto que en nuestra investigación además aporta a la aplicación del programa Escritura Creativa influye en la Presentación textual en el desarrollo de la capacidad de producción de textos narrativos en los estudiantes del $4^{\circ}$ grado de la Institución Educativa de Primaria $\mathrm{N}^{\circ} 22238$ del Provincia de Chincha 2015.

Gallego y García (2010), señala que Leer y escribir en la escuela III: La comprensión y producción de textos narrativos en el grado tercero del Instituto técnico, al manejar una propuesta didáctica partiendo de las necesidades de los estudiantes, se logra cubrir en un alto porcentaje esas necesidades; se tiene en cuenta los intereses de los estudiantes, se dan elementos que permitan que ellos retomen lo que tienen en su ambiente para llegar a la creación de historias, que poco a poco a nivel cognitivo y desde el desarrollo de las habilidades antes mencionadas, esa estructuración paulatina permite superar esas falencias dadas en los procesos de escritura y de lectura, que se identificaron al principio de la intervención, en nuestra investigación podemos decir que el programa de Escritura Creativa influye en el Proceso de la escritura de textos narrativos en los estudiantes del $4^{\circ}$ grado de la Institución Educativa de Primaria $N^{\circ} 22238$ provincia de Chincha 2015.

Buitrago (2009), en su investigación el Diseño e implementación de un ambiente virtual del aprendizaje que fomenta la producción de textos narrativos en estudiantes del sexto grado de la Institución Educativa Ana Josefa Morales Duque, en Santander de Quilichao, Cauca. Bogotá destaca el objetivo de diseñar e implementar un ambiente virtual para fomentar la producción de textos narrativos en estudiantes de sexto grado, objetivo que implicó el cumplimiento de una secuencia de pasos: Desarrollo de un fundamento teórico para el diseño de un ambiente virtual de aprendizaje dirigido a la producción de textos narrativos, en nuestra investigación podemos decir que la aplicación del programa Escritura Creativa influye en producción de textos narrativos en los estudiantes del $4^{\circ}$ grado de la
Institución Educativa de Primaria No 22238 Provincia de Chincha 2015.

De la Cruz (2009), en su tesis doctoral "Las estrategias de producción de textos narrativos y su evaluación en el desarrollo de las capacidades de comunicación integral en los alumnos del $\mathrm{V}$ ciclo de Educación Básica regular de la Institución Educativa José Pardo y Barreda de Chincha, se llegó a las siguientes conclusión: La aplicación de estrategias en la producción de textos Narrativos mejoran las capacidades de redacción y creación en los alumnos del quinto grado de educación básica de la Institución Educativa Pública José Pardo y Barreda de Chincha, en nuestra investigación el programa de Escritura Creativa influye en las capacidades del uso de la normativa en los estudiantes del $4^{\circ}$ grado de la Institución Educativa de Primaria No 22238 Provincia de Chincha 2015.

Finalmente, después de haber rrealizado las discusiones con otras estudios sobre las dimensiones se sugiere al Ministerio de Educación capacite a los docentes del nivel primario en la aplicación del programa Escritura Creativa para con la finalidad que los estudiantes mejoren el aprendizaje de la producción de textos narrativos, que les servirán a los estudiantes para ser más creativos, tengan la facilidad de escribir y obtener buenos aprendizajes significativos.

\section{AGRADECIMIENTOS}

A los estudiantes del $4^{\circ}$ grado de la Institución Educativa $N^{\circ} 22238$, a sus docentes, autoridad edil de Chincha Baja, a mi asesor doctor Abelardo Campana Concha y al director de la revista Científica Infinitum...por haber contribuido desinteresadamente en la realización de la presente investigación.

\section{REFERENCIAS BIBLIOGRAFICAS}

Beaugrande, R. D. (1981). Les contraintes générales qui affectent les processus de compréhension du langage in Langage et compréhension. Bulletin de Psychologie Paris, 35(356), 683-694.

Buitrago, N. (2009). Diseño e implementación de un ambiente virtual del aprendizaje que fomenta la producción de textos narrativos en estudiantes del sexto grado de la Institución Educativa Ana Josefa Morales Duque, en Santander de Quilichao, Cauca. Bogatá.

Campos, V. \& Mariños, Z. (2009). Influencia del programa "Escribe cortito, pero bonito" en la producción de textos narrativos: mitos, cuentos y leyendas del área de comunicación de los/las estudiantes de 
cuarto grado de primaria de la IE N 088005 "Corazón de Jesús" de Chimbote - Ancash 2009. Tesis para obtener el grado de Magister en Educación. Universidad Cesar Vallejo, Chimbote, Perú.

Cassany, D. (2003). La escritura electrónica. Cultura y educación, 15(3), 239-251.

Cassany, D. (1990). Enfoques didácticos para la enseñanza de la expresión escrita. Comunicación, lenguaje y educación, 2(6),

De La Cruz, M. (2009). "Las 63-80.estrategias de producción de textos narrativos y su evaluación en el desarrollo de las capacidades de comunicación integral en los alumnos del $\mathrm{V}$ ciclo de Educación Básica regular de la Institución Educativa José Pardo y Barreda de Chincha" Perú.

Fuentes Sanhueza, J. A. (2008). Estudio experimental del discurso narrativo escrito: un modelo de intervención didáctica en el aula. Tesis para obtener el grado de Magister en didáctica de la lengua materna. Universidad del Bío-Bío. Concepción, Chile.

Gallego, L., \& García, Y. (2010). Leer y escribir en la escuela III: La comprensión y producción de textos narrativos en el grado tercero del instituto técnico superior. Trabajo de grado para optar el título de Licenciada en pedagogía Infantil. Universidad Tecnológica de Pereira. Pereira, Colombia.

Gardner, H. (1995). Mentes creativas. Barcelona: Paidós.

Lomas, C. (1999). Cómo enseñar a hacer cosas con las palabras: teoría y práctica de la educación lingüística. Barcelona: Paidós.

Correo electrónico: vilmahuamanle@hotmail.com

Revisión de pares:

Recibido: 02/11/2019

Aceptado: 20/12/2019 